ARTÍCULOS ORIGINALES Rev Chil Salud Pública 2013; Vol 17 (3): 237-244

\section{ABORDAJE CUALITATIVO SOBRE LA INCORPORACIÓN DEL ENFOQUE DE SALUD INTERCULTURAL EN LA MALLA CURRICULAR DE UNIVERSIDADES CHILENAS RELACIONADAS CON ZONAS INDÍGENAS}

A QUALITATIVE APPROACH FOR INCORPORATION OF INTERCULTURAL HEALTH IN THE CURRICULUM OF CHILEAN UNIVERSITIES IN REGIONS WITH LARGE INDIGENOUS POPULATIONS.
Adalguisa Painemilla Hospital Makewe Universidad de Chile adalpainemical@gmail. com

Gabriel Sanhueza Escuela de Salud Pública Universidad de Chile

JaIro VANEGAS Escuela de Salud Pública Universidad de Chile

\section{RESUMEN}

En Chile, la Salud Intercultural es un tema emergente. Existen alrededor de 13 pueblos originarios, de los cuales solo ocho están reconocidos y valorados por la Ley Indígena 19.253. Estos son los aymara, quechua, atacameño, rapanui, koya, kawésqar, yamana y mapuche. A su vez, en estas áreas existen universidades públicas y privadas que ofrecen diferentes carreras en el área de la salud, preparando diferentes recursos humanos en estas zonas. Por tanto, la incorporación de la salud intercultural en la malla curricular de las universidades resulta relevante. Este estudio cualitativo se plantea conocer si la salud intercultural está o no incorporada dentro de la malla curricular en las instituciones del Consejo de Rectores de Universidades Chilenas que imparten carreras de la salud, como enfermería, kinesiología, medicina, nutrición y obstetricia en ocho universidades en las regiones primera, novena, decimoquinta y Metropolitana. Solo una universidad de ellas tiene incorporado el enfoque Salud Intercultural. Algunas universidades contemplan este enfoque en los objetivos de los programas aunque su desarrollo como concepto y como práctica es insuficiente.

\section{ABSTRACT}

In Chile, the Intercultural Health is an emerging issue. There are about thirteen peoples, of which only eight are recognized and valued by the 
Indigenous Law 19.253. These are the Aymara, Quechua, Atacama, Rapa Nui, Koya, Kaweshkar, Yamana and Mapuche. In turn, these areas are public and private universities that offer different careers in health human resources preparing different in these areas. Therefore, the incorporation of intercultural health in the curriculum of universities is relevant. To learn about this, he raised a qualitative study to know whether or not incorporated into the curriculum or programs of study in the institutions of the Council of Rectors of Chilean Universities who provide bealth careers such as nursing, kinesiology, medicine, Nutrition and Obstetrics in 8 universities in Regions I, IX, XV and Metropolitan Region. In conclusion, only one university has incorporated the Intercultural Health approach. Some colleges provide for this focus on program objectives is still insufficient development as a concept and practice.

\section{INTRODUCCIÓN}

Décadas atrás los estados latinoamericanos realizaron cambios que han permitido el reconocimiento constitucional de su carácter multiétnico y pluricultural $(1,2)$. En la década pasada se dió inicio a incorporación en los censos poblacionales preguntas para determinar el porcentaje de individuos pertenecientes a los pueblos originarios. De 15 países que realizaron censos en el año 2000, 13 de ellos incorporaron alguna pregunta basada en autorreferencia o lenguaje materno (3). También, se han planteado dificultades de carácter metodológico que no permite dimensionar la importancia demográfica y su impacto en la formulación de políticas públicas con enfoque intercultural $(4,5)$. Diferentes estudios epidemiológicos siguen demostrando la existencia de inequidades y desigualdades sociales en Latinoamérica, siendo los grupos étnicos uno de los más vulnerables.

Respecto a lo interculturalidad en salud, Ibacache (1997) sostiene que es la capacidad de actuar en equilibrio entre el conocimiento, las creencias y prácticas culturales diferentes respecto a la salud y la enfermedad (6). La enfermedad es una experiencia particular de cada cultura. Por tanto, lo que se percibe como salud enfermedad está determinado por cada cultura específica $(7,8)$. Por tanto, tiene que ver con la relación entre dos modelos con dos sistemas médicos distintos de formación pero que deben tratar de coexistir (9). Esto hace importante la promoción del análisis sociocultural de los sistemas de salud con enfoque intercultural en las estrategias de curación, rehabilitación, prevención y promoción de la salud (10).

Por su parte, la OPS (1999) afirma que los centros académicos que forman recursos humanos en salud no incluyen en sus programas de estudio el enfoque intercultural de forma continua, lo que conduce a limitaciones para entender el contexto sociocultural de la población que va a ser atendida. Dentro de las universidades de América Latina que imparten posgrado en salud intercultural se encuentra las siguientes: Universidad Indígena Intercultural (UII), Universidad Tomás Frías de Potosí y la Universidad de las Regiones Autónomas de la Costa Caribe Nicaragüense (URACCAN) y las Universidades Indígenas Mexicanas (11-14).

En Chile, la Salud Intercultural es un tema emergente e importante de abordar debido a la diversidad cultural existente. Hay alrededor de 13 pueblos originarios, de los cuales solo ocho están reconocidos y valorados por la Ley Indígena 19.253. Estos son: aymara, quechua, atacameño, rapanui, koya, kawésqar, yamana y mapuche. De estos, los aymara representan el $7 \%$ y los mapuche un $87,3 \%$ 
(Censo 2002). Durante el 2001 se dieron las bases para la creación de la política indígena de "Nuevo Trato con los Pueblos Indígenas, la Sociedad Chilena y el Estado”. En materia legislativa Chile ha planteando la Ley Indígena 19.253 del año 1993. Además se han creado instancias tales como, Corporación Nacional de Desarrollo Indígena (CONADI), (15-18), Programa Tolerancia y No discriminación, Plan Nacional para Superar la Discriminación en Chile 2001-2006 $(19,20)$ y en el ámbito de la salud, el Ministerio de Salud (MINSAL) reconoce a los agentes de salud indígena y coordina trabajos con el sistema público de salud (21-23).

Respecto a la educación, el Estado chileno la define como el proceso multidireccional mediante el cual se transmiten conocimientos, valores, costumbres y formas de actuar (24). Una importante innovación son las mallas curriculares que muestran los contenidos y hacen trasparentan una determinada carrera o áreas disciplinares. En Chile existe un gran número de universidades, tanto públicas como privadas. Las públicas integradas en el Consejo de Rectores de las Universidades Chilenas (CRUCH) (25). Al referirse a "diversidad cultural” la historiadora Fanny López V. señala: "El sistema educativo como mediador en la construcción de identidad de quienes asisten a ellas, han sido espacios donde la reflexión desde y sobre lo cultural ha sido relegada a un segundo plano y fragmentada, convirtiéndose en un asunto excluyente" (26).

La formación de recursos humanos se ha considerado un eje prioritario del plan nacional de salud, señalándose que las instituciones formadoras deben poseer capacidad de abordar la diversidad cultural y la implementación de nuevos paradigmas. También, se señala que en la formación de pregrado y posgrado se debe incorporar el enfoque intercultural para crear capacidades y responder al perfil y necesidades reales de las comunidades indígenas $(11,27)$.

Debido a lo anterior, los autores plantean un estudio con enfoque cualitativo respecto a Salud Intercultural, con el propósito de conocer la incorporación en la malla curricular o programas de estudio de las instituciones que integran el Consejo de Rectores de Universidades Chilenas que imparten carreras de la salud como: Enfermería, Kinesiología, Medicina, Nutrición y Obstetricia en 8 universidades en las regiones primera, novena, decimoquinta y Metropolitana.

\section{Material y método}

Este estudio es de carácter cualitativo, con dos componentes. El primero, es un análisis documental y el segundo, una entrevista semiestructurada auxiliada por el método de análisis de contenido. La revisión documental se desarrolló en las universidades y las entrevistas se aplicaron a los directores y jefes de los diferentes programas de estudios. En total participaron 8 universidades y 23 funcionarios entre académicos y administrativos, ubicadas en Tarapacá, Araucanía, Arica Parinacota y en la región Metropolitana. Estas universidades se caracterizan por estar ubicadas en zonas de alta concentración de población indígena: Universidad Arturo Prat (I y IX Región), Universidad de Chile (Región Metropolitana), Universidad de la Frontera (IX Región), Universidad Metropolitana de Ciencias de la Educación, Pontificia Universidad Católica de Chile (Metropolitana), Universidad de Santiago de Chile (Metropolitana) y Universidad de Tarapacá (XV Región).

La revisión documental abordó los sitios web y los programas de estudios elaborados a los cuales se accedió con el permiso correspondiente de cada universidad. Se extrajeron textualmente los contenidos y objetivos que incluían Salud Intercultural. Esta revisión se realizó entre los meses de octubre a diciembre del año 2008 y enero del 2009. Las preguntas de las entrevistas estaban relacionadas con salud y pueblos indígenas, y conocimiento de la medicina tradicional. Estas fueron grabadas y para garantizar la privacidad de los entrevistados, se asignó una sigla a cada uno de ellos, de acuerdo a la carrera que representaron y la posición o lugar que les correspondió al realizar la entrevista. A partir de estos resultados se generaron diferentes categorías de análisis (Ver tabla). 
Tabla 1. Categorías y Subcategorías del Análisis de las Entrevistas.

\begin{tabular}{ll}
\hline CATEGORIAS & SUBCATEGORIAS \\
\hline Conceptode Salud Intercultural & - Relación entre culturas diferentes \\
& - Visión de salud-enfermedad de las distintas culturas \\
Experiencias de Salud Intercultural en la & - Integración de salud intercultural a la educación de profesionales de la salud \\
Formación de las Universidades & - Valoración e integración de la medicina tradicional indígena a la educación \\
& de profesionales de la salud \\
Motivación en la Incorporación de Salud & - Necesidad sociocultural \\
Intercultural a las Mallas Curriculares de & - Mejora en la calidad de la atención \\
las Carreras de la Salud & - Beneficios para la promoción de salud \\
& - Fortalecimiento vínculo profesional -paciente \\
Experiencia de Salud Intercultural (SI) en en & - Significado de la Incorporación de SI en los servicios públicos de salud \\
Regiones & - Beneficios en los servicios de salud con la incorporación de SI en las \\
& mallas curriculares \\
\hline
\end{tabular}

\section{RESULTADOS}

\section{Revisión documental: Presencia de salud in- tercultural en las mallas curriculares dentro de la formación}

Se observó que la única universidad que incorpora en sus programas el tema de Salud Intercultural es la Escuela de Enfermería de la Pontificia Universidad Católica de Chile, como una asignatura obligatoria en la carrera de Licenciatura en Enfermería con una equivalencia de diez créditos, que además es impartida como ramo electivo para otras carreras. Dentro de esta asignatura se abordan áreas como antropología y sociología, dictadas en el primer o segundo año.

La carrera de Medicina y Nutrición de la Universidad de Chile y la Universidad de La Frontera ofrecen seminarios relacionados con Medicina tradicional / alternativa y alimentación tradicional mapuche. Esta última ofrece un diplomado internacional a distancia en Salud Intercultural.

La Universidad de Santiago y la Universidad de Chile, abordan la salud intercultural y cosmovisión mapuche así como la preparación de facilitadores que trabajan en programas de salud intercultural. La Universidad Arturo Prat, de Iquique, ofrece cursos "Electivos de Formación General” relacionados con la cosmovisión aymara, integración social en la diversidad, lengua, visión de mundo y comunicación. La Universidad de Tarapacá (primera región), ofrece un curso sobre "Lengua y Cultura Aymara hacia una Educación
Bicultural”. El resto de las universidades de las regiones en estudio no registran ningún tipo de programa formal relacionado al tema de la interculturalidad en salud.

\section{Respecto a la revisión de los programas o planes de estudio}

La Pontificia Universidad Católica de Chile muestra entre sus contenidos antropología, sociología, respuestas culturales de la salud y enfermedad, donde se integran: la medicina occidental, la tradicional y alternativas, la medicina mapuche y la salud intercultural. La mayoría de las carreras han integrado la salud intercultural o parte de ella tales como: determinantes socioculturales, cultura como determinante de salud, concepto salud-enfermedad, aspectos psicológicos y etnia.

Las carreras que incluyen salud intercultural o parte de ella, están representadas a través de categorías y subcategorías. Para el análisis, se seleccionaron solo algunos contenidos y objetivos, debido a que eran semejantes entre sí (saturación de la información). Dentro de las carreras analizadas y a excepción de la carrera de Enfermería de la Pontificia Universidad Católica de Chile (PUC), las demás han incluido de alguna u otra manera en sus asignaturas la salud intercultural.

\section{Entrevistas a informantes clave}

Concepto de salud intercultural: Los funcionarios entrevistados no tenían claro el concepto, por lo que construían su propia 
definición. Entre los aspectos que incluyeron fue "la relación entre culturas diferentes o la visión que estas tienen sobre salud".

Relación entre culturas diferentes: Los entrevistados relacionan la cultura indígena con la definición de salud de la OMS, refiriéndose solo al tema de salud, no a la interacción de dos medicinas diferentes, aunque mencionan el aspecto cultural.

Visión de salud-enfermedad de las distintas culturas: Los entrevistados tienen diferentes formas de entender y abordar este concepto. Se afirma que se necesita lograr el bienestar de las personas, las familias y la comunidad, pero que en algunas ocasiones se ve dificultada por la barrera comunicacional entre las diferentes culturas. Sin embargo, el concepto de los pueblos indígenas, incluye además, la naturaleza, la espiritualidad, relación entre seres humanos y el medio ambiente.

Integración de salud intercultural a la educación de profesionales de la salud: Algunos suponen que la mayoría de las carreras de la salud han incorporado estos conceptos, pero al parecer no están seguros de esto. Uno de los entrevistados afirma que la integración de la salud intercultural no se encuentra aún dentro de la malla curricular; sin embargo, al revisar el programa se puede observar que sí se menciona. Se reconoce que se está en una fase inicial, siendo más fácil seguir haciendo lo cotidiano que promover el cambio. Se puede afirmar que no existe un trabajo concreto y en conjunto. Debido al desconocimiento del Programa de Salud Intercultural, algunos de los entrevistados/as la confunden con las terapias complementarias o alternativas.

Valoración e integración de medicina tradicional indígena a la educación de profesionales de la salud: Se entiende a través de los entrevistados/as que existe poco interés en (re)conocer la medicina tradicional indígena. Para algunos entrevistados, las universidades que le han dado el espacio a la medicina tradicional indígena dentro de la formación de profesionales les ha permitido desarrollar proyectos de investigación.

Motivación en la incorporación de salud intercultural a las mallas curriculares de las carreras de la salud. A través de esta categoría se mencionan algunos aportes que contribuyen a la incorporación de la interculturalidad en salud a las mallas curriculares, representado por la expresión, motivación o valoración que los directores/as y/o jefes/ as de las carreras de Enfermería, Kinesiología, Medicina, Nutrición y Obstetricia de las Universidades Chilenas del Consejo de Rectores entregan con respecto a su integración.

Necesidad sociocultural: Los directores señalan que la salud intercultural ha sido un elemento importante en la realización de los diagnósticos de salud; prueba de ello es que se ha incorporado en varios cursos. Sin embargo, lo anterior no se encuentra explicitado por escrito en los programas formativos.

Fortalecimiento vínculo profesionalpaciente. Este permite al profesional poder relacionarse y entender de manera más integral a su paciente. Fortalece su relación, permitiendo una mayor adherencia por parte del paciente. El Sistema de Salud y el profesional de salud deben comprender las diferentes realidades para dar una atención de calidad.

Experiencia de salud intercultural en regiones: La primera experiencia obtenida fue en el hospital Makewe-Pelale junto al Servicio de Salud Araucanía Sur, de la novena región. Sin embargo, los entrevistados afirman que el vínculo que existía entre la Universidad de La Frontera y este hospital se ha perdido.

Significado de la incorporación de la salud intercultural en los servicios públicos de salud: Se señala que se ha tenido un buen avance, porque se está respetando la visión de otras culturas frente a lo que es salud, permitiendo de esta manera que los procedimientos logren una mayor adherencia. Además, permite conocer y valorar al otro, ya que si no existe el reconocimiento no existe la valoración.

Beneficios en los Servicios de Salud con la incorporación de salud intercultural en las mallas curriculares: Los entrevistados lo valoran positivamente, porque los futuros profesionales tendrían una visión más amplia en la atención de los pacientes, permitiendo una mayor participación de la gente, y con ello el aumento de la accesibilidad a la atención en salud, permitiendo realizar mejores diagnósticos con la participación del propio. 


\section{DISCUSIÓN}

A pesar de que la Organización Panamericana de la Salud (OPS) han abordado la problemática de salud de los pueblos indígenas $(10,12)$, en Chile los principales centros de formación aún no la incluyen en sus planes de estudios como asignatura, a excepción -como se señalara- de la Escuela de Enfermería de la Pontificia Universidad Católica de Chile (12).

Algunas de las universidades han incorporado este tema a las asignaturas de antropología y de sociología, impartidas en los primeros niveles de formación de los profesionales de la salud, y principalmente en la carrera de Medicina y Kinesiología de la Universidad de Chile y de las carreras de Enfermería y Obstetricia del resto de las universidades, donde los contenidos y objetivos guardan relación con la diversidad cultural existente (1), enfoques diferentes de salud-enfermedad, medicina de los pueblos originarios, especialmente del pueblo aymara y mapuche, y sus respectivos especialistas tradicionales $(11,12,28,29)$. No se observaron asignaturas que incorporaran un taller práctico, en el que se realicen visitas a los centros de salud que trabajan con el programa de salud intercultural.

A pesar de que salud intercultural es parte del Programa de Salud del Ministerio de Salud (MINSAL) (19), esto es desconocido por algunos entrevistados. Primero, por considerar que solo se circunscribe al área de la araucanía y la zona norte y segundo, porque se confunde la medicina alternativa con la medicina tradicional indígena.

La motivación de algunas de las universidades a integrar la salud intercultural a los programas de estudios tiene que ver con el trabajo que lleva realizando el Ministerio de Salud con los pueblos indígenas $(14,16,30)$, aunque la mayoría de los directores/as de las universidades de la región Metropolitana lo desconoce. Pese a ello, se ha ido introduciendo lentamente, con excepción de la Escuela de Enfermería de la Pontificia Universidad Católica de Chile, donde se imparte (4, 19, 20). Su incorporación permitiría una mejor adhesión de los pacientes con sus tratamientos, evitando así las recidivas y el abandono, sobre todo cuando las patologías son de larga data o infectocontagiosas.

Una de las motivaciones mencionadas por directores/as o jefes/as de las carreras de salud, es el lograr mejorar la calidad de la atención, señalando que con la incorporación de la salud intercultural a las mallas curriculares o programas de estudio se respondería eficiente y eficazmente a las demandas de salud de la población (14). Los entrevistados reconocen también que los pacientes serían vistos en forma más integral, considerando los factores sociales y culturales, donde predominaría el aspecto biopsicosocial y no solo el biomédico.

Finalmente, se pudo constatar que el programa de salud intercultural es poco conocido en el ámbito de la formación de los profesionales de la salud, no así las medicinas alternativas. Por lo tanto, si se considera esto, se puede decir que la incorporación de la salud intercultural a las mallas curriculares o a los programas de estudios ha sido mínima, y lo que se ha logrado es debido a la iniciativa de los propios docentes, especialmente en las asignaturas de las ciencias sociales, y en algunos casos de algunos de los directores/as o jefes/as de las carreras en estudio y de los propios estudiantes.

En conclusión: la única universidad que incorpora en sus programas el tema de salud intercultural es la Escuela de Enfermería de la Pontificia Universidad Católica de Chile. Las carreras que han incorporado salud intercultural o parte de ella en sus programas son enfermería y obstetricia. Los programas de formación de las carreras de enfermería, kinesiología, medicina, nutrición y obstetricia no han incorporado la salud intercultural en su malla curricular. Sin embargo, existen algunas que la incorporan como objetivos o contenidos, especialmente en las relacionadas con las ciencias sociales, como antropología y sociología. En relación con los contenidos, estos se encuentran presentes y relacionados con interculturalidad a través del concepto de salud-enfermedad, donde se incluyen algunos aspectos de los pueblos originarios del país, especialmente su cultura y cosmovisión. Respecto de la motivación y experiencia de 
las/os directores de las carreras de enfermería, kinesiología, medicina, nutrición y obstetricia, la investigación permite afirmar que como programa es un tema que no se conoce lo suficiente y que no se le ha dado mayor énfasis en la preparación de recursos humanos en salud. Sin embargo, se reconoce que con la incorporación de la interculturalidad dentro de las mallas curriculares o programas de estudios, se obtendría una mejora en la calidad de la atención, se ampliaría la visión de resolución de problemas de salud y el vínculo profesional-paciente aumentaría. Asu vez, esto lograría una mejor adherencia a los tratamientos y mayor participación del propio paciente, su familia y la comunidad.

\section{REFERENCIAS}

1. Bello A. Etnicidad y ciudadania en América Latina. La acción colectiva de los pueblos indigenas. Santiago, Chile: CEPAL. 2004.

2. Boccara G. The mapuche people in postdictatorship Chile, Études rurales. 2002; 3(163-164): 283-303.

3. Comisión Económica para América Latina. Pueblos indígenas y afrodescendientes de América Latina y el Caribe: Información sociodemográfica para políticas y programas. CEPAL. 2005. Santiago de Chile.

4. Schkolink S, Del Popolo F. Los censos y los pueblos indígenas en América Latina: una metodología regional. Seminario Internacional. Santiago: abril 27-29.05. CEPAL. 2005.

5. Chackiel J, Peyser A. La población indígena en los censos de América Latina. En: Estudios sociodemográficos de pueblos indígenas. Santiago: CELADE-CIDOB-FNUAPICL. 1994.

6. Ibacache J. La Salud, el desarrollo y la equidad en un contexto intercultural [en línea]. Chile: Servicio de Salud Araucanía Sur; 1997 [consultado 21.08.2007]. Disponible en: http://www.mapuche.info/mapuint/ibaca00.htm

7. Citarella L. Medicina y cultura en la Araucanía. Santiago: Editorial Sudamericana. 1995.
8. Alarcon A, Vidal A, Neira J. Salud intercultural: Elementos para la construcción de sus bases conceptuales. Rev Med Chile. 2003; 131: 1061-1065.

9. Hernández Cassiani R. Instituto de Educación e Investigación Manuel Zapata Olivella: una educación intercultural para reafirmar las diferencias. En: Daniel Mato (Coord). Diversidad cultural e interculturalidad en educación superior: Experiencias en América Latina. Caracas: Instituto Internacional de la UNESCO para la Educación Superior en América Latina y el Caribe (UNESCOIESALC): 255-262.

10. Organización Panamericana de la Salud. $A r-$ monización de los sistemas de salud indígenas y el sistema de salud convencional en las Américas: lineamientos estratégicos para la incorporación de las perspectivas, medicinas $y$ terapias indígenas en la atención primaria de salud [en línea]. Washington, D.C.: OPS. 2003 [consultado agosto 2007]. Disponible en: http://www.paho.org/Spanish/AD/ THS/OS/INDI49esp.doc

11. Rosell Puig W, Más García M. El enfoque sistémico en el contenido de la enseñanza [en línea]. La Habana: Instituto Superior de Ciencias Médicas de La Habana. Facultad de Ciencias Medicas "Dr. Enrique Cabrera”. 2003 [consultado 26.08.2008]. Disponible en: http://bvs.sld.cu/revistas/ems/ vol17_2_03/ems02203.htm

12. Universidad Andina Simón Bolivar. Bolivia: UASB [en línea] [consultado 4.11.2009] Disponible en: http://www.uasb.edu. bo/portal/index.php?option=com content\&view $=$ article $\&$ id $=8$

13. Segunda Reunión de la Subred de Salud Intercultural Quito-Ecuador; $10 \mathrm{y}$ 11.09.09. [consultado 4.11.2009]. Disponible en: http://new.paho.org/hq/ index2.php?option $=$ com_content\&do_ $\mathrm{pdf}=1 \& \mathrm{id}=1831$

14. Lafuente López R. Sobre el análisis y representación de documentos [en línea]. México: Centro Universitario de Investigaciones Bibliotecológicas de la UNAM. 2001 [consultado 5.11.2009]. Disponible en: http:// www.ejournal.unam.mx/ibi/vol15-30/ IBI03009.pdf 
15. Batzin C. El desarrollo bumano y los pueblos indígenas. Seminario Internacional; CEPAL; 27 al 29.04.05. Santiago de Chile: CEPAL. 2005.

16. Ser Indígena. Programa de la ONG Comunidad Ser Indígena. Portal de las culturas originarias de Chile. http://www. serindigena.org/territorios/aymara/pay_01. htm [consultado 8.11.2007].

17. Real Academia Española. Diccionario de la Lengua Española. 22a edición. España: Espasa Calpe. 2001.

18. Chile. Ley 19.253. Establece Normas sobre Protección, Fomento y Desarrollo de los Indígenas, y Crea la Corporación Nacional de Desarrollo Indígena.

19. Chile. Ministerio de Salud. Resolución exenta 91. Políticas de salud y pueblos indígenas. Chile: MINSAL. 2006.

20. Miembros Red Multicultural. Miembros Red Ministerial. Bases Generales del Plan Nacional para Superar la Discriminación en Chile. [Santiago de Chile]: Gobierno de Chile [2001?].

21. Ibacache J, Chureo F, McFall S, Quidel J. Promoción de la medicina y terapias indígenas en la atención primaria de salud: el caso de los mapuche de Makewe-Pelale [en línea]. Washington, D.C.: OPS. 2001 [consultado 20.08.2007]. Disponible en: http://www. paho.org/Spanish/HSP/HSO/indig16_esp. doc

22. Chile. Ministerio Secretaría General de Gobierno. Política de nuevo trato con los pueblos indigenas: Derechos Indigenas, Desarrollo con Identidad y Diversidad Cultural [en línea]. Santiago, Chile: MSGG. SECC. 2004 [consultado el 20 Agosto 2007]. Disponible en: http://www.ministeriodesarrollosocial.gob.cl/admin/docdescargas/centrodoc/ centrodoc_168.pdf.

23. Mays V, Ponce N, Washington D \& Cochran S. Classification of race and ethnicity: Implications for public health. Ann Rev Public Health. 2003; 24: 83-110.

24. Chile. Ministerio de Educación. Glosario de términos [en línea] [consultado 3.11.2009].
Disponible en: http://w3app.mineduc.cl/ Genero/glosario_de_terminos

25. Consejo de Rectores de las Universidades Chilenas. Santiago: CRUCH [en línea] [consultado el 18.07.2008]. Disponible en: http://www.consejoderectores.cl/web/

26. López Valencia F. La investigación intercultural en la formación de jóvenes universitarios [en línea]. En: Rebetez Motta N, Ganduglia Néstor G (Coords). El descubrimiento pendiente de América Latina: diversidad de saberes en diálogo hacia un proyecto integrador. Montevideo: Signo UNESCO - Universidad de La Rioja. 2005; pp. 157-166 [consultado 18.07.2008]. Disponible en: http://dialnet.unirioja.es/servlet/ articulo codigo $=1335626 \mathrm{v}$

27. El concepto de competencia en pedagogía conceptual [en línea] [consultado 26.08.2008]. Disponible en: http://www. monografias.com/trabajos23/competenciapedagogia/competencia-pedagogia.shtml

28. Cunningham M. Evolución, tendencias y principales características de la educación superior indígena en Nicaragua, a partir de la experiencia de URACCAN. En: UNESCO-IESALC (Comps). La educación superior indígena en América Latina. Caracas: Instituto Internacional de la UNESCO para la Educación Superior en América Latina y el Caribe (UNESCOIESALC). 2003; pp. 171-195.

29. Bolaños G, Libia T, Avelina P. Universidad autónoma, indígena e intercultural: un espacio para el posicionamiento de epistemologías diversas. En: Mato D (Coord). Diversidad cultural e interculturalidad en educación superior: experiencias en América Latina. Caracas: Instituto Internacional de la UNESCO para la Educación Superior en América Latina y el Caribe (UNESCOIESALC). 2008; pp. 211-222.

30. Vázquez M, Ferreira M. Análisis de los datos cualitativos. En: Introducción a los métodos cualitativos de investigación aplicados en salud. X Escuela Internacional de Verano 2008. Santiago: Universidad de Chile. 2008. 